\title{
Critique de la dématérialisation
}

In: Communication et langages. N¹40, 2ème trimestre 2004. pp. 55-68.

\section{Résumé}

La notion de « dématérialisation » constitue actuellement l'une des pièces maîtresses de l'idéologie de la communication. Or, après enquête, Pascal Robert constate que la dématérialisation ne se rencontre ni dans les TIC ni dans les réseaux où se dévoilent en revanche un nouveau mode de matérialisation ainsi qu'un processus de virtualisation par changement d'échelle. L'auteur s'interroge donc sur la fonction sociale et politique que cette notion joue dans les discours qui la convoquent (économistes, gestionnaires, juristes, secteur des TIC, libertaires, voire critiques) et avance l'hypothèse selon laquelle la dématérialisation sert à la fois à justifier les acteurs et à soustraire les TIC aux épreuves de justification (politiques, économiques) susceptibles d'incarner la figure du réel, moyen pratique de soutenir l'abstraction idéologique.

Citer ce document / Cite this document :

Robert Pascal. Critique de la dématérialisation. In: Communication et langages. N140, 2ème trimestre 2004. pp. 55-68.

doi : 10.3406/colan.2004.3268

http://www.persee.fr/web/revues/home/prescript/article/colan_0336-1500_2004_num_140_1_3268 


\section{Critique de la dématérialisation}

Les autoroutes de l'information ne sont rien d'autre que le déplacement à l'échelle mondiale de bits sans poids à la vitesse de la lumière (N. Negroponte, L'homme numérique, Pocket, 1995, p. 24)

Nous allons vivre dans un monde nouveau : basse friction (B. Gates, op. cit, 1995, p. 195)

Ici il n'y a pas de matière (J.P. Barlow, op. cit, 1996)

Les images photographiques, vidéographiques ou holographiques (...) sont des images matérielles (...). En revanche, les images idéelles, infographiques, ne sont pas matérielles, sauf par accident (P. Quéau, Le virtuel, Champ Vallon, 1993, p. 176)

Contempteurs comme thuriféraires de la société de l'information se retrouvent facilement dans l'idée que l'un des effets les plus fondamentaux de nos technologies de l'information et de la communication consiste en une véritable dématérialisation ${ }^{1}$. On le regrette ou on s'en réjouit. Mais dans tous les cas la dématérialisation est prise comme un fait, une évidence qui, en tant que telle ne demande aucune explication. C'est justement une telle évidence que

1. Nous devons à Y. Jeanneret une première approche critique de cette notion lorsqu'il s'insurge contre la supposée dématérialisation du texte. Cf. Y a-t-il (vraiment) des technologies de l'information?, Presses du Septentrion, 2000, p. 68-79 et 114-117. Il a récemment étendu, avec E. Souchier, sa critique à l'économie et la culture supposées dématérialisées en soulignant que «la notion d'immatériel rend très mal compte de ce qui est en jeu et masque l'importance des propriétés des supports, le poids des contextes matériels d'utilisation et le pouvoir des formes imposées par les inscriptions, les matrices et les cadres de la communication ", Cf. Y. Jeanneret et E. Souchier, 2002, " La communication médiatisée estelle un "usage" "?, Communication \& langages, $\mathrm{n}^{\circ} 132$, p. 20.
TECHNOLOGIE

\section{PASCAL ROBERT}

La notion de "dématérialisation" constitue actuellement l'une des pièces maîtresses de l'idéologie de la communication. Or, après enquête, Pascal Robert constate que la dématérialisation ne se rencontre ni dans les TIC ni dans les réseaux où se dévoilent en revanche un nouveau mode de matérialisation ainsi qu'un processus de virtualisation par changement d'échelle. L'auteur s'interroge donc sur la fonction sociale et politique que cette notion joue dans les discours qui la convoquent (économistes, gestionnaires, juristes, secteur des TIC, libertaires, voire critiques) et avance l'hypothèse selon laquelle la dématérialisation sert à la fois à justifier les acteurs et à soustraire les TIC aux épreuves de justification (politiques, économiques) susceptibles d'incarner la figure du réel, moyen pratique de soutenir l'abstraction idéologique. 
cette réflexion voudrait interroger. Car ses implications se révèlent considérables, notamment sur le plan idéologique et politique.

En effet, dès lors que l'on suppose cette dématérialisation possible, elle ouvre sur un monde qui s'oppose directement et radicalement à celui dont nous sortirions, à savoir, inversement, un monde tout entier saisi par la matière. C'est donc, a priori, voir l'ancien bien concret et le nouveau bien abstrait. Sous sa forme canonique, le matériel renvoie chez un N. Negroponte, aux atomes, à ce qui pèse, ce qui est lourd et encombrant. Bref, la matière, le matériel, c'est ce qui entrave le déplacement, ce qui frotte et freine le mouvement, c'est quelque chose dans lequel on s'englue, on s'enferme ou qui nous engloutit. Cette matière-là n'a rien à voir avec celle des anciens, celle qui engendrait la forme, celle qui, mater, mère, s'offrait comme potentiel de formes qu'un élément mâle révélait dans l'application d'un geste viril! Non, cette matière que l'on condamne le plus souvent - et surtout les apôtres de la société de l'information - colle comme boue aux semelles et empêche l'élévation, l'envol, déçoit toute tentative d'accession à cette légèreté - à laquelle nous aspirerions tant - qui nous libérerait des contraintes de l'espace et de ses objets. Car voilà l'ennemi : l'espace encombré d'objets qui exige du temps pour les déplacements des hommes ou des choses, qui les ralentit alors même qu'ils sont déjà plombés par le poids et le volume de leurs propres amas d'atomes.

Levez ces contraintes, dès lors tout est possible : tout glisse, tout s'évapore dans une abstraction qui rend les métamorphoses, les combinaisons, les déplacements singulièrement faciles, tout se reproduit et se duplique, rien ne coûte, rien n'est pénible, etc. S'évanouit donc par là même le travail - un travail dont le destin est par définition complètement lié à celui de la matière, de son ouvrage, de sa mise en forme, à la sueur des corps. La dématérialisation c'est l'ouverture à toutes les pseudo-utopies, à tous les possibles ou plutôt les impossibles : elle devient le nom qu'il convient d'invoquer si l'on veut porter cette révolution économique et politique de la nouvelle société. Elle constitue ainsi une matière première de choix dans les montages idéologiques les plus divers. Puisque la notion aborde un ailleurs, un futur, d'autres modes de faire, il est dès lors possible de la convoquer pour élaborer des montages discursifs susceptibles de lever les épreuves de justification qui ont cours ici et maintenant et donnent la forme de leur réalité sociale aux hommes, à leurs valeurs et à leurs objets ${ }^{2}$. Soit, en effet, elle crédibilise ce qui n'est pas encore, ou ce qui fonctionne selon des lois que l'on ne connaît pas encore; avec le radicalement nouveau qu'elle emporte elle clôt toute épreuve de justification par sa seule présence, sa seule évidence : c'est nouveau ! Soit, plus paradoxalement peut-être, elle permet de raccrocher au passé sans et afin de ne pas penser les différences : le droit s'applique tel quel diton, les lois de l'économie aussi.

Nous allons dans un premier temps partir à la recherche de cette dématérialisation, puis, rentré bredouille de notre exploration, nous nous interrogerons dans un second temps sur les fonctions sociales que remplit une telle notion.

2. Nous nous inspirons ici de Laurent Thévenot et Luc Boltanski, 1991, De la justification, Gallimard. 


\section{À La RECHERCHE de La dématérIALISATION}

\section{Dans les mémoires?}

Auparavant, inscrit sur le papier, le texte restait lisible a priori-constamment disponible à la lecture; avec l'informatique/numérisation le texte (ou l'image) est enfoui dans la mémoire - c'est-à-dire au sein même de l'outil de mémorisation, disquette, disque dur, CD-Rom ou DVD peu importe. C'est pourquoi il exige un instrument de lecture qui le restitue sous une forme appréhendable directement par un esprit humain : en l'occurrence ces codes de représentation que l'on nomme écriture (alphabétique ou non). Cette restitution vaut comme ensemble de traductions qui permettent de faire remonter si l'on veut l'information de son codage primordial - dans la logique informatique - du binaire - illisible pour l'homme - à cette écriture façonnée par des siècles, voire des millénaires de pratique. Alors même que le texte couché sur papier "flotte " à sa surface et peut, par là même être saisi au vol, l'information sous forme binaire, à la fois réduite - par ce codage alternatif de base et considérablement étendue - car un énoncé quelconque, dès lors qu'il est traduit dans ce langage requiert incomparablement plus de 0 ou de 1 que de lettres de l'alphabet par exemple ! - est directement en prise avec la matière, inscrit non plus seulement sur une matière, mais bien avec l'état (et les changements d'états) même de la matière. Autrement dit, nos nouvelles mémoires ne sont en rien dématérialisées et moins encore immatérielles : elles relèvent simplement d'un nouveau « mode de matérialisation » qui, non seulement est au moins aussi matériel, mais peut-être plus encore que le précédent.

Ce qui change le plus c'est d'une part que l'inscription ne suit plus directement le geste de la main, elle s'affranchit de la trace laissée par le scripteur (outil et homme) sur une surface, et d'autre part (et les deux sont liés) l'échelle du phénomène d'inscription : il ne s'effectue plus à échelle d'homme, mais bien à celle de la machine - ce que l'on peut appeler, si l'on veut, un processus de "virtualisation ». Que nous ne percevions plus le processus d'inscription, que nous n'ayons plus une prise directe, musculaire, mécanique sur lui, ne peut en aucun cas signer à la fois sa disparition et celle de sa dimension matérielle. L'information ne s'en trouve pas dématérialisée, mais virtualisée, en ce sens qu'elle n'est plus directement accessible à nos sens parce qu'elle est engluée dans la matière, littéralement matérialisée.

\section{Par la reproductibilité ?}

La reproduction du document a passé pendant longtemps - et passe encore - par la vieille machinerie de l'imprimerie. Certains ont pu croire que cette imprimerie permettait déjà d'opérer une distinction de fond entre matériel et immatériel : la multiplication des formes sous lesquelles le texte se donnerait à lire le libérerait en quelque sorte comme texte pur, comme réalité purement informationnelle, immatérielle. Or, la reproduction orchestrée par l'imprimerie correspond tout au contraire à une multiplication des formes matérielles sous lesquelles le texte s'offre à la lecture. Autrement dit, quelque multiplié que soit un texte ou même ses différentes versions, il n'en va aucunement d'un effet de dématérialisation, mais bien plus simplement d'un foisonnement de ses modalités d'existence matérielle. Non seulement il n'en va pas d'un processus d'immatérialisation, mais jamais il n'y a eu 
autant de matière, cette inflation de papiers que nos bibliothèques parviennent décidément moins à maîtriser qu'elles ne s'époumonent à la suivre.

Pas plus que l'imprimerie, l'informatique n'atteint une quelconque dématérialisation. Au contraire même, jamais le codage n'aura été à ce point piégé par la matière même - ne grave-t-on pas un CD-ROM ? En ce sens la reproduction renvoie moins à une improbable absence de matière qu'à une multiplication des supports et des formes matérielles. Qu'un même fichier puisse être affiché à travers un écran ou sur un papier n'est en rien une régression de son degré de matérialité, mais montre au contraire les différents modes de sa matérialisation: qui ne sont rien moins qu'au nombre de trois (disque, écran, papier). Dès lors, comprendre le passage d'une matière (papier) à l'autre (disque) non comme une multiplication de supports, mais comme un découplage média/support ne peut s'expliquer que par la nécessité pour certains acteurs de monter une stratégie d'argumentation susceptible de légitimer ou d'éviter d'avoir à justifier une prise de position quelconque - au sens où ils investissent cette position afin de défendre leurs intérêts ( $c f$. la deuxième partie, "Les fonctions sociales de la notion de dématérialisation $»)$.

\section{Dans/par le réseau ?}

On pourrait nous objecter que le réseau, lui, produit bien un effet de dématérialisation... sauf à oublier la matérialité de l'infrastructure du réseau lui-même, de ce que nous avons appelé ailleurs la logistique informationnelle ${ }^{3}$. Là encore il en va d'un changement d'échelle qui nous rend les choses difficiles à appréhender : de même que dans l'infiniment petit de l'inscription de l'information dans la matière du disque on en vient à oublier cette matière, dans l'infiniment grand - à nos yeux, comme à l'aune de notre imagination - du réseau de réseaux, l'on oublie, symétriquement, tous les atomes qu'il convient de convoquer pour faire circuler et mémoriser des bits. Avoir accès à un site Web hébergé sur un serveur éloigné de quelques milliers de kilomètres, ne consacre une dématérialisation qu'à condition d'appréhender la technique sous une forme purement magique : là encore, l'échelle de temps, la vitesse, est telle que nos ordres de grandeurs traduisent cette quasi-instantanéité (pas toujours effective) par une absence : absence d'infrastructure, absence de matière, absence de réelle circulation, absence de temps - alors même que le temps n'a pas disparu, mais que l'unité de temps a changé. Une unité que nos sens ne sont pas - pas encore ? - équipés à traiter. Leur grille temporelle ne peut retenir ces unités temporelles beaucoup trop fines, elles n'en recueillent dans ses mailles qu'un agglomérat grossier, inévitablement décalé. Facette temporelle de ce processus - singulièrement déroutant

3. Pascal Robert, "La logistique informationnelle, réseaux, information, valeur et pouvoir ", Médiaspouvoirs, nouvelle série, $n^{\circ} 3,2^{e}$ trimestre 1998, p. 127 à 144. Cette logistique informationnelle repose aujourd'hui sur de gigantesques réseaux qui mêlent inextricablement technique, gestion et politique, les "macro-systèmes techniques " ou large technical systems. Sur l'application aux TIC d'une telle approche, $c f$. Pascal Robert, «Les autoroutes de l'information au miroir de la problématique des macro-systèmes-techniques », Terminal, n 71-72, L'Harmattan, Paris, 1996, p. 33 à 70 . "Vers une déstabilisation des macro-systèmes-techniques?, le travail des technologies de l'information et de la communication », Flux, n³6-37, ENPC/CNRS, Paris, 1999, p. 16 à 22. «La typologie des MST au miroir des TIC ", Flux, n 55, ENPC, Paris, 2004, p. 53 à 58. 
- du changement d'échelle - et que nous appelons ici « virtualisation ». Le découplage ne s'opère pas entre média et support, entre information et matière, mais bien entre nos sens, nos ordres de grandeurs et les échelles spatiales et temporelles auxquelles fonctionnent nos TIC : un découplage qui exige de nous - collectivement, sociétalement, un effort de recalibrage de nos ordres de grandeurs spatiotemporels. On peut regretter d'être soumis à une telle injonction, on ne peut pour autant la récuser d'un revers de main, sans avoir à en subir de lourdes conséquences.

\section{Dans les effets des TIC ?}

Peut-être va-t-on enfin repérer cette dématérialisation dans ce que l'on peut appeler les effets des TIC. Car si les TIC sont effectivement immatérielles, alors elles ne sont pas censées engendrer des effets typiques de l'économie matérielle, telle que la pollution. Deuxième test : si les TIC sont réellement immatérielles alors elles ne peuvent par définition pas plus participer d'un effet de "matérialisation » de services restés jusque-là plutôt abstraits.

Des TIC immatérielles peuvent-elles polluer sur le même mode que des technologies supposées matérielles? La réponse ne peut être théoriquement que négative ; car si elle était positive cela prouverait qu'elles sont loin d'être véritablement immatérielles et que l'on verse dans l'abus de langage. Immatérielles, de telles TIC ne pourraient en aucune manière générer des déchets ni produire une quelconque pollution de type chimique notamment. Or, des études ont montré qu'il n'en est rien : les déchets, bien physiques, biens matériels, sont considérables et leur capacité à engendrer une pollution chimique également ${ }^{4}$. Les atteintes à l'environnement peuvent aussi se révéler plus indirectes : les TIC, prises globalement et comptables en millions d'ordinateurs par exemple, consomment beaucoup d'électricité. Or produire une telle électricité - que ce soit en brûlant une énergie fossile telle que le charbon ou en ayant recours au nucléaire - emporte un coût écologique non négligeable. Quant à la consommation de papier - qui entraîne les conséquences que l'on sait sur la déforestation, alors que l'industrie de la pâte à papier est l'une des plus polluantes - non seulement elle ne régresse pas, mais elle ne cesse d'augmenter - le bureau sans papier reste bien, en l'état actuel des choses, une vue de l'esprit.

Si les TIC étaient vraiment immatérielles, pourraient-elles donner lieu à des processus de production d'" effets de matérialisation " ? Or, il existe de nombreux services, ou plutôt un nombre considérable d'opérations qui se trouvent en leur cœur, qui, dès lors qu'ils sont produits et transitent sur des réseaux TIC ne manquent pas de produire de tels effets. Nous prendrions volontiers la bourse comme exemple symptomatique. En effet, la bourse a toujours été un lieu certes concret mais dont les opérations restaient pour le moins abstraites. Le recours massif aux TIC dans le fonctionnement même des marchés boursiers emporte une véritable mise en visibilité de leurs opérations. Sur les écrans d'ordinateurs les tableaux et graphiques mettent véritablement en scène ce grand 
60 technologie

ballet de l'information boursière. Les opérations acquièrent ainsi une visibilité sémiotique nouvelle 5 .

D'une manière plus globale encore, le Web met en accès et donne à voir des documents qui, sans lui, resteraient beaucoup moins potentiellement visibles. Il ne s'agit pas de se leurrer sur une supposée égalité de l'accès de (et non pas seulement a) l'information sur internet - les logiques de hiérarchisation et de filtrage sont déjà à l'œuvre -, mais de constater que tel document qui n'avait aucune chance de pouvoir être connu sans le Web, peut éventuellement posséder une petite chance de l'être avec lui. La différence est certes faible et la probabilité infime, mais elles sont bien réelles dans les deux cas. Or, l'immatériel donne-t-il à voir? Ne faut-il pas encore et toujours un support - bien matériel - pour rendre une telle opération possible - quand bien même l'exposition est découplée de l'enregistrement et quand bien même ce que l'on voit est le produit d'une virtualisation.

Bref, on ne constate aucune dématérialisation tout simplement parce qu'il n'y a pas d'information sans support ! Ce qui laisse à penser que le thème de la dématérialisation n'a peut-être rien à voir avec les TIC, mais par contre, beaucoup avec les acteurs ou les idéologies qui l'instrumentalisent.

\section{LES FONCTIONS SOCIALES DE LA NOTION DE DÉMATÉRIALISATION}

Les ardents défenseurs de la société de l'information comme ses farouches opposants ont besoin de cette dématérialisation soit parce qu'elle justifie tout, pour les uns, soit parce qu'on la stigmatise comme, justement, ce qui justifie tous les excès, pour les autres! Bref, personne ne tient à la récuser et n'y a intérêt.

\section{L'économiste, le gestionnaire et le juriste}

Les économistes, les gestionnaires et les juristes sont particulièrement exposés. Certains économistes pourraient en effet être fortement tentés d'agiter cette dématérialisation comme preuve de la scientificité de leur discipline, à un moment où le scepticisme règne, jusque dans leurs rangs ${ }^{6}$. La démonstration reposerait sur le raisonnement suivant : si les principes de la science économique restent valables alors même que change le monde, que l'on passe d'un univers dominé par la matière à un univers dématérialisé, alors cela prouve leur validité. C'est-à-dire qu'ils montrent par là même leur universalité. La science économique aurait alors tout intérêt à promouvoir cette notion de dématérialisation, comme preuve de sa scientificité. D’où une position à son égard qui risquerait de relever parfois plus de la pétition de principe que de l'enquête. Heureusement nos collègues restent prudents et le plus souvent ne s'engagent pas dans la qualification du monde sur lequel ouvrent les TIC - il n'en va ni d'une dématérialisation ou d'une immatérialisation, ni d'un nouveau mode de matérialisation - simplement :

«la technologie change. Les lois de l'économie restent " ?.

5. Cf. Knorr Cetina, Karin et Bruegger, URS, « La technologie habitée. La forme de vie globale des marchés financiers ", Réseaux, $\mathrm{n}^{\circ} 122,2003$.

6. Et pose directement question au contenu même de l'enseignement de la science économique, cf. Alternative économique, $\mathrm{n}^{\circ} 187$ (2000) et $\mathrm{n}^{\circ} 196$ (2001).

7. Carl Shapiro et Hal Varian, Economie de l'information, De Boeck, 1999, p. 8. 
Mais, à bien y regarder, le raisonnement ne reste-t-il pas au fond similaire? Car l'évolution frénétique de la technique ne modifie pas plus que l'immatériel les supposées "lois de l'économie ». C'est dire que la capacité même de l'économie à expliquer - à principes constants - les changements du monde, prouve sa scientificité, la validité universelle de ses principes. Introduire un ouvrage - très intéressant par ailleurs - consacré à l'"économie de l'information " par un tel postulat n'est-ce pas s'interdire a priori de penser, justement, que l'information et ses changements de supports, peuvent ne seraitce qu'interroger, questionner sur un mode heuristique, les principes fondamentaux de la science économique?

Une même tentation ne guette-t-elle pas les gestionnaires? Certains d'entre eux voient ainsi des investissements immatériels partout, " puisque tout ou presque dans une organisation tend à devenir immatériel ${ }^{8}$; d'autres n'hésitent pas à titrer que «le management est mort, vive le e management » ${ }^{9}$. Or, qu'y a-t-il de singulièrement immatériel dans de la recherche-développement - se ferait-elle sans laboratoires ni instruments? -, ou même dans des logiciels dont on ne voit pas véritablement à quoi ils peuvent servir s'ils ne tournent pas dans des machines ni ne donnent à voir, par une interface quelconque, l'information qu'ils portent et traitent ? La notion d'investissement de forme promue par Thévenot n'était-elle pas plus judicieuse ${ }^{10}$ ? Cela dit, alors que dans le $n^{\circ} 129$ de la Revue Française de gestion M. Kalika et $\mathrm{H}$. Isaac parlent de e management et "d'entreprise numérique " ${ }^{11}$ sans faire référence aux notions d'immatériel et de dématérialisation, mais en mobilisant celle de "virtualisation " ${ }^{12}$, le Krach internet semble revaloriser dans le numéro suivant $\left(\mathrm{n}^{\circ} 130\right)$ la notion vaste et floue d'immatériel : une notion qui certes dépasse les TIC mais dont on peut se demander si elle ne risque pas de devenir le cadre de leur appréhension ? N'est-ce pas ce qui incite R. de Rochebrune à écrire que :

« les bienfaits évidents de cette avancée technologique (internet), que notre récent dossier sur le e management tout comme l'ensemble des recherches sur l'immatériel que contient ce numéro prouvent si nécessaire en matière de gestion, ne pourront que mieux apparaître dans un tel contexte apaisé ${ }^{13}$ (post-krach) ?

L'évidence technologique justifie ainsi l'évidence gestionnaire - et réciproquement. Or, la logique de l'évidence repose sur un véritable paradoxe, car soit ce dont on parle (l'énoncé) est réellement évident et il n'est pas besoin de le souligner

8. Ahmed Bounfour, introduction au dossier consacré à l'immatériel : « Une nouvelle approche de la gestion ", Revue Française de gestion, n 130, Septembre-octobre 2000, p. 93.

9. Michel Kalika, «Le management est mort, vive le e-management », Revue française de gestion, $\mathrm{n}^{\circ} 129$, Juin-juillet-aout 2000.

10. Laurent Thévenot, 1986, «Les investissements de forme ", in Thévenot (éd.), Conventions économiques, Cee-PUF, p. 21-71.

11. Henri Isaac, "L'entreprise numérique », Revue française de gestion, $n^{\circ} 129,2000$.

12. Qui ne fait d'ailleurs pas l'objet d'une définition précise et est volontiers confondue avec la numérisation.

13. L'éditorial du $\mathrm{n}^{\circ} 130$ de la Revue franfaise de gestion, op. cit. 
(énonciation), soit on estime devoir affirmer cette évidence, dans un geste qui, tout à la fois, en avoue la défaillance et la compense en posant l'évidence de la chose (énonciation) plutôt que la chose dans son évidence même (énoncé), afin d'éviter d'avoir à se poser quelque question ou devoir y répondre. Cette divergence entre les deux niveaux de l'énoncé et de l'énonciation consacre justement ce que l'on appelle un paradoxe ${ }^{14}$. Si la phrase de Rochebrune est vraie, il n'a aucunement besoin de l'écrire, elle efface d'elle-même sa propre nécessité ; s'il l'écrit c'est justement qu'il n'y a pas d'évidence et que cette absence ouvre l'espace potentiel d'un questionnement dont cette seule pétition de principe (de l'existence même d'une supposée évidence des bienfaits d'internet) empêche l'actualisation.

Enfin, le juriste lui-même saura-t-il résister aux sirènes de cette dématérialisation? Car le droit est singulièrement déstabilisé par la montée en puissance des TIC. Aussi les juristes tendent-ils à adopter deux positions :

- soit ils partent du principe que le droit s'applique, mais il doit alors justifier son intervention; autrement dit, cette intervention ne possède plus l'automaticité implicite d'une évidence, il lui est nécessaire de réaffirmer haut et fort sa continuité. Ainsi, explique le Conseil d'État, «Internet et les réseaux numériques, c'est avant tout un nouvel espace (...). Cet espace n'est pas naturellement celui du droit ${ }^{15}$ (p. 13). Néanmoins, quelque neuf pages de justification plus loin, il affirme : "il apparaît que les questions juridiques suscitées par le développement d'internet et des réseaux numériques ne sont pas de nature à remettre en cause les fondements mêmes de notre droit "(p. 22). Dans la même veine M. Bibent soutient que :

" considérée dans sa valeur économique, l'information est un bien. D'une nature certes particulière, ce bien informationnel est immatériel. Cette caractéristique n'est pas nouvelle pour le droit qui considère depuis longtemps qu'un bien immatériel peut être objet de droit, pourvu qu'il soit considéré comme tel économiquement. Le développement des TIC (...) ne lui confere aucun caractère fondamentalement différent ${ }^{16}$ (p. 6).

Ainsi le droit réaffirme-t-il (énoncé) sa continuité sur et malgré des mondes hétérogènes, soutient-il la validité de ses principes dans un univers de l'immatériel (le "nouvel espace" du $\mathrm{CE}$ ), alors même que ce besoin trahit son manque d'évidence (énonciation).

- soit le droit se sait déjà subordonné à et donc reconfiguré par internet, les réseaux et les TIC : " tout notre droit devra être repensé en conséquence " ${ }^{17}$ (p. 122). Un droit qui doit encore plus se justifier tout en sachant que même la notion d'immatériel/dématérialisation n'empêchera pas qu'il chute de son piédestal ! Mais cette justification ne passe plus par la démonstration de l'applicabilité de ses principes sur un

14. Daniel Bougnoux, 1998, Introduction aux sciences de l'information et de la communication, La découverte, Paris, p. 26.

15. Conseil d'État, Internet et les réseaux numériques, La documentation française, 1998.

16. Michel Bibent, Le droit du traitement de l'information, ADBS/Nathan, 2000.

17. André Bertrand et Thierry Piette-Coudoul, Internet et le droit, PUF, 1999. 


\section{Critique de la dématérialisation $\mid 63$}

terrain différent, mais de l'intérêt que peut avoir la technique d'accepter, malgré tout, son offre de service en matière de régulation. Ce n'est bien évidemment pas signer la fin du droit, mais d'un droit susceptible de se saisir et de maîtriser l'objet technique - gestation ou avènement en revanche d'un droit contre-dépendant de cet objet technique.

En deçà de cette dernière position qui entérine le cas - encore extrême d'une vassalisation, nous sommes bien dans un montage que j'appelle "l'impensé ": un discours, qui fonctionne de facto comme un outil de justification, se présente comme la baguette magique dont l'intervention permet de faire l'économie d'une épreuve de justification ${ }^{18}$. Paradoxe donc: l'énoncé " dématérialisation " fonctionne comme un instrument de levée des épreuves de justification, alors que son énonciation fonctionne comme justification dans l'épreuve même qu'il cherche à voiler.

\section{Le secteur des TIC}

Lorsque l'on aborde le secteur économique des TIC, la dématérialisation joue également un rôle de dispense de justification. En effet, si le monde d'internet est un monde dématérialisé, dans lequel les contraintes habituelles d'espace, de temps, de coûts d'accès, d'apprentissage, etc. sont annulées ou à tout le moins largement atténuées, alors tout devient possible : et d'abord « faire de l'argent » rapidement et facilement. Comme le trompette $\mathrm{B}$. Gates lui-même (qui a compris qu'il ne suffisait pas de vendre des TIC, mais qu'il fallait également vendre l'idéologie qui va avec et les porte) : " nous allons vivre dans un monde nouveau : basse friction, frais généraux peu élevés, information pléthorique et opérations peu coûteuses. Le paradis de l'acheteur ${ }^{19}$ (Gates 1995, p. 195). Or, quoi de plus dématérialisé et tranquille qu'un "paradis »?

D'où cette attitude moins que moyennement responsable de ces sociétés de financement, de ces officines de capital-risque qui ont parié sur une multitude de projets sans pour autant s'être véritablement assurées d'un contrôle qualité des dossiers de faisabilité. Autrement dit, alors que le montage d'un projet requiert de son porteur de passer traditionnellement un nombre important d'épreuves, testant tant ses qualités personnelles que celles de son dossier, " on " a cru pouvoir s'en dispenser dans le cadre de ce nouvel univers dématérialisé d'internet. La dématérialisation justifiait ainsi l'absence de toute prudence élémentaire. Les propriétés du nouveau monde de la dématérialisation ne pouvaient, supposait-on, que donner un jeu enfin gagnant-gagnant. Sidéré par cet espace informationnel, on en est venu à négliger de s'informer sur les candidats et la fiabilité de leur proposition. On a également largement surestimé la valeur des entreprises leaders du secteur : là encore la dématérialisation, qui ouvrait sur une nouvelle frontière, devait les dispenser de justification. L'espace informationnel dématérialisé valait

18. Pascal Robert, «Confiance, technique et justification, le rôle du macro-techno-discours informatique dans l'établissement d'un climat de confiance ", Quaderni n ${ }^{\circ} 46,2002$, p. 53-66 ; Pascal Robert, "Le bogue de l'an 2000, un antidote à l'impensé informatique?, confiance risque et technique ", Terminal $\mathrm{n}^{\circ} 82,2002$, p. 107-122.

19. Bill Gates, La route du futur, Pocket, 1995, p. 195. 
en quelque sorte auto justification; justification par la logique de l'évidence : ça ne peut que marcher puisque c'est sur internet !

L'entreprise n'était pas évaluée en fonction de ses actifs et de ses résultats, mais seulement en fonction d'un avenir qui, dématérialisé, ne pouvait être que souriant. Où l'on supposait à la dématérialisation la vertu d'un multiplicateur : propriété supposée intrinsèque à ce nouvel espace. Ce qui n'avait guère de valeur dans l'ancien monde, au regard des critères d'évaluation de cet ancien monde, pouvait très bien valoir beaucoup dans le nouveau, puisque, par définition il n'était supposé aucune continuité entre les deux. D'où le gonflement d'une bulle dans l'évaluation de la capitalisation boursière de ces sociétés. Où l'idéologie de la communication - singulièrement performative ici - prend les vecteurs privilégiés de son développement à son propre piège.

Le retour du réel s'effectue dès lors que l'on commence à ré-embrayer sur les épreuves de justification habituelles : ces sociétés gagnent-elles de l'argent - c'està-dire leurs actionnaires peuvent-ils en gagner ? Ces projets rencontrent-ils des clients convaincus en suffisamment grand nombre? La publicité est-elle efficace sur le net?, etc. Retour sur le plancher des vaches des " lois de l'économie " - facilement confondues avec le simple bon sens - soulignent nos économistes qui pensent trouver là, quant à eux, le passage réussi d'une épreuve de justification qui conforte leur science. Ce qui les dispensera de chercher de nouveaux principes susceptibles d'expliquer ce qui se passe sur cet espace d'un nouveau mode de matérialisation. Où il est à craindre qu'en définitive l'on se satisfasse malgré tout de la notion de dématérialisation.

\section{Le libertaire et le critique}

John Perry Barlow a proposé aux internautes une "Déclaration d'indépendance " sur le mode, très américain, de celle qui a enclenché le mouvement de détachement de la colonie du nouveau monde de l'empire britannique. Et à lire Barlow le parallèle a sa logique, car internet constitue bien à ses yeux un "nouveau monde », un monde où les

« notions juridiques de propriétés, d'expression, d'identité, de mouvement et de contexte ne s'appliquent pas à nous. Elles se fondent sur la matière. Ici, il n'y a pas de matière ${ }^{20}$ (p. 51).

Où l'on aborde donc les rivages d'un espace où règnent les « bien immatériels $»^{21}$ (p. 86), passibles $d^{\prime}$ " échanges non physiques » (p. 90) parce que "la technologie numérique détache l'information de son support » (p. 86). Cette dernière remarque mérite que l'on s'y arrête, parce qu'elle suppose que si un même support peut faire circuler divers types de "médias " (texte, données, images, sons, etc.) il faut en conclure qu'il autorise l'indépendance de cette information par rapport au support : une telle conclusion n'est-elle pas pour le moins quelque peu sollicitée? Auparavant, en effet, un même support subissait une

20. John Perry Barlow, « Déclaration d'indépendance », in Libres enfants du savoir numérique, L'Éclat, 2000, p. 47-55.

21. John Perry Barlow, «Vendre du vin sans les bouteilles : l'économie de l'esprit sur le réseau global », in Libres enfants du savoir numérique, l'Éclat, 2000, p. 83-129. 
sorte de spécialisation fonctionnelle, qui lui permettait de donner à voir ou à entendre un seul type de média : le papier privilégiait le texte, le Celluloïd l'image, l'onde la voix, etc. Or, aujourd'hui la numérisation, nous l'avons vu, ne libère pas l'information du support. En revanche elle affranchit le support non de l'information, mais d'un seul type de mise en forme, d'un seul type de média si l'on veut, parce qu'il peut les porter et les combiner tous.

Barlow en vient même à affirmer que :

« les bouteilles physico-numériques auxquelles nous sommes accoutumés, telles que les disquettes, les CD-ROM et autres emballages distincts de bits conditionnés sous plastique, vont disparaître avec le branchement de tous les ordinateurs sur le réseau global (...). On pourrait rétorquer à cela que l'information requerra encore un élément physique, tel qu'une existence magnétique sur les disques durs titanesques de lointains serveurs, mais ce sont là des bouteilles qui n'ont aucune forme macroscopiquement distincte ou personnelle significative" (p. 88-89).

Bref, plus la technique devient macrosystémique, plus elle gonfle et s'amplifie, plus elle se complexifie et plus elle disparaît, s'invisibilise ! Il s'agit selon la métaphore de Barlow de "vendre du vin sans les bouteilles » : c'est pourquoi nous assistons à ce tour de prestidigitation où les macrobouteilles, parce qu'elles ne sont plus visibles en tant que telles, voire peut-être ne sont plus pensables en tant que telles, s'évaporent littéralement, et l'épreuve de justification avec elles. Or, c'est aussi ce dispositif macrosystémique qu'il convient justement d'essayer de penser malgré tout, malgré sa taille et sa complexité - et avec lui les risques d'autonomisation d'un système technique que nous ne maîtrisons peut-être déjà plus et que nous ne maîtriserons peut-être que de moins en moins ${ }^{22}$. Certes, Barlow croit en un internet utopique. Mais cet internet, s'il peut contrebalancer celui des marchands, peut tout aussi bien alimenter se son eau naïve le moulin libéral. Bref, penser en terme de dématérialisation semble considérablement abaisser les capacités critiques.

Ainsi en va-t-il de la cryptographie dont Barlow prétend qu'elle « est le matériau dont seront composés les murs, les frontières - et les bouteilles du cyberespace » (p. 122) : où, donc, Barlow lui-même nous apprend que les fonctions d'enfermement, de définition de frontières, ne vont pas disparaître du cyberespace, mais seulement être exercées autrement - en l'occurrence grâce à la cryptographie. Or, ce pouvoir cryptographique ne joue pas dans les sphères éthérées d'une supposée immatérialité, mais bien avec et dans le tissu même de la nouvelle matière numérique - dont il convient de garder constamment à l'esprit qu'elle ne tient et ne peut s'exprimer que par le truchement des systèmes techniques bien concrets qui la soutiennent. Le droit lui-même, s'il doit, selon Barlow, disparaître sous la forme actuelle, subsistera comme fonction de régulation, mais désormais activée par la technique elle-même, incorporée et mise en œuvre au sein même de "l'architecture du net " (p. 116) - au " terrain » (p. 116) comme le dit Barlow. "Terrain », son lapsus (?) n'est-il pas révélateur d'un retour du refoulé, la matière, pour désigner, justement, ce qui est censé advenir 
sur les conditions même de son exil ? Dès lors la liberté d'expression n'a plus à faire l'objet d'une garantie constitutionnelle, puisqu'elle évolue dans un " environnement qui (...) traite la censure comme un dysfonctionnement " (p. 117) : vision étroitement fonctionnaliste/machinique moins promesse de liberté, nous semble-t-il, que de dépendance à l'égard d'un macrosystème technique en voie d'autonomisation. Enfin, la cryptologie, elle aussi censée nous protéger contre les intrusions intempestives des puissants (gouvernements ou entreprises) n'introduit-elle pas une logique de défiance généralisée qui va à l'encontre même d'un véritable régime de liberté en démocratie ? Car celle-ci repose sur une confiance a priori en l'autre qui nous incite à ne pas nous promener tous armés (un principe dont le non-respect mine à la base la démocratie américaine), ni à blinder nos courriers mais seulement à les glisser sous une enveloppe somme toute légère.

La dématérialisation fonde une logique technicienne qui repousse toujours plus loin les épreuves de justification qui pourraient lui être opposées. Elle liquéfie les réseaux qui l'emportent, aussi vastes, complexes et réellement physiques soient-ils; et avec eux le droit comme marqueur de la propriété privée. Ne reste, dans une telle perspective, qu'un tissu vivant de communications qu'il convient de protéger par la cryptographie. Acceptez la dématérialisation et tout s'ensuit, sans laisser le moindre interstice à la lourde machine de la justification.

Barlow est un amoureux du net et comme tous les amoureux, il le rêve - or en amour le réveil est parfois brutal. Le critique, lui, n'est pas là pour rêver, mais pour dévoiler ce que les idéologies au service des puissants nous cachent. Or, les "critiques » de la société de l'information ne manquent pas qui soulignent à l'envi que la dématérialisation est la mère de tous nos maux - comme, symétriquement, elle est mère de toutes joies pour ses thuriféraires. Elle n'est pas à comprendre, mais à dénoncer : c'est pourquoi elle doit, effectivement, pour eux aussi posséder toutes ces propriétés que lui reconnaissent ses défenseurs, et singulièrement cette capacité à supprimer les contraintes qui pèsent si lourdement sur l'ancien monde, c'est-à-dire sur le réel. Car, pour certains critiques, toute épreuve de justification ne peut être montée qu'à partir de cet ancien monde qui est le seul dont nous disposions comme référence et qui, dès lors est investi de la lourde mission de s'assumer comme figure du « réel ». Penser en terme de modes différents de matérialisation comme nous tentons de le faire ici, permet, justement, de sortir de cette logique d'opposition radicale et stérile ; à nos yeux le «monde-hors-du-réseau » n'est pas plus ni moins réel que celui du réseau. Notre société a inventé un nouveau mode de matérialisation qu'il nous revient de comprendre, et non de récuser ou d'encenser a priori. À l'inverse, le critique qui reste piégé par la dématérialisation l'utilise pour monter une fausse épreuve de justification : il a justement besoin de cette dématérialisation comme repoussoir, comme ennemi, comme base de tous les excès. C'est bien pourquoi il ne cherche à, ni n'envisage de la critiquer en tant que telle. Elle n'est en rien ce qui est à critiquer, mais au contraire, dans son discours, ce qui fonde la critique elle-même : son existence est l'une des conditions primordiales de la critique, elle la justifie - et le critique avec elle. La dématérialisation est au critique ce que 


\section{Critique de la dématérialisation $\mid 67$}

le moulin est à Don Quichotte : ce contre quoi il se bat, ce qu'il récuse, mais qui, également, justifie son action !

\section{LA DÉMATÉRIALISATION : UNE NOTION POLITIQUE}

La dématérialisation justifie ainsi à la fois, l'économiste, le gestionnaire, le juriste, l'investisseur aussi bien que le libertaire et même le critique : parce qu'elle est tout terrain, parce qu'elle peut être instrumentalisée, enrôlée dirait un B. Latour, au service des causes les plus diverses on ne peut que lui prédire un avenir prometteur! Elle est un de ces points de cristallisation discursive autour duquel l'idéologie de la communication peut organiser le réel. Cette idéologie de la communication travaille justement à l'évitement des épreuves de justification - ce qu'on loue ou regrette (dans le cas du critique). Ce qui résistait et qui, donc exigeait des efforts pour être maîtrisé, c'est-à-dire du travail, obéit au doigt et à l'œil, ce qui était difficile devient facile, ce qui prenait beaucoup de temps relève de l'instantané, l'espace s'efface dans la foulée... ou ce qui était matière devient immatériel. Bref, tout ce qui était épreuve que l'on pouvait opposer à celui qui se prétendait a priori compétent, comme test, justement, de son savoir-faire, ne se pose plus comme problème, s'évanouit : ne reste que des solutions aime-t-on dire, simples, pratiques, rapides, n'exigeant aucun effort, aucune dépense d'énergie. La dématérialisation participe de ce monde enchanté où s'abolit le travail.

Mais l'idéologie de la communication est une fusée à deux étages : car la levée des épreuves de justification d'un coté permet de pratiquer par ailleurs cette même opération, mais sans avoir à le reconnaître. Où l'on retrouve notre économiste et notre critique : ils sont entièrement agis par cette idéologie de la communication dans leur instrumentalisation de la dématérialisation (positive ou négative) puisqu'elle leur permet de monter, de fait, une épreuve de justification qu'ils remportent avec succès et qui démontre ainsi leur compétence - dans le silence le plus total. Il convient donc de rappeler encore une fois la structure profondément paradoxale de cette idéologie de la communication qui à la fois, contribue au montage d'un discours de dispense, évitement ou contournement des épreuves de justification - au bénéfice de la communication et/ou d'une TIC, alors qu'il constitue de facto un outil de justification qui disparaît en quelque sorte derrière sa propre opération. Un discours qui dispense de la justification n'est pas supposé être lui-même un discours de justification; or, c'est bien ce qu'il est en pratique, parce qu'il ne peut pas ne pas en être un! C'est pourquoi la dématérialisation permet à l'économiste par exemple de se justifier de facto dès lors qu'il la présente ou l'accepte comme ce qui ouvre un espace où les barrières de la justification (les contraintes habituelles en terme d'espace/temps et de coûts notamment) sont abaissées au maximum. C'est pourquoi, symétriquement, certains critiques se justifient eux aussi par la même opération - à la connotation négative près.

Cette notion de dématérialisation fonctionne comme l'un des outils du montage d'une opération politique. En effet, dès lors qu'un univers est supposé dématérialisé, il autorise et facilite certains glissements de sens : c'est ainsi que certaines fonctions traditionnellement étiquetées comme politiques - à l'image de la frontière - peuvent faire l'objet d'un arrêté de dissolution a priori - la dématérialisation équivaudrait à l'absence même de toute barrière. Autrement dit, elle 
permet de partir du principe - non contesté, non interrogé - d'une incompatibilité radicale entre ce nouveau monde et la frontière. Or, nous avons montré ailleurs en détail qu'il n'en est rien ${ }^{23}$. D'ailleurs il suffit de modifier l'angle de lecture, de récuser la dématérialisation, l'immatériel, de considérer qu'il en va pratiquement d'un nouveau mode de matérialisation pour comprendre que ce dernier peut très bien accueillir et mettre en œuvre, même si c'est dans des formes éventuellement différentes, la même fonction - en l'occurrence la fonction-frontière, qui repose sur le tri et le classement, opérations qu'autorise également le nouveau mode de matérialisation. Construire une incompatibilité radicale entre l'immatériel et une fonction politique, alors même qu'elle n'existe pas, permet de fait, de réinstaurer l'exercice d'une telle fonction - en catimini si l'on veut - au bénéfice d'acteurs qui pourront se croire dispensés de l'acquisition de la légitimité politique (qui, dans nos démocraties relève d'un processus de sélection public, long et difficile, au sein d'une pluralité et/ou de l'appartenance à la bureaucratie de l'état légal-rationnel). Ce que nous appelons le glissement de la prérogative politique, régression de la gestion politique de notre société telle que nous essayons de la construire depuis la fin du Moyen âge. Il ne s'agit bien évidemment pas d'une fin du politique, mais de la modification de sa forme par une perte de légitimation - démocratique - de la souveraineté. Autrement dit, la fonction politique serait directement reliée à - dans le sens ou elle ouvrirait sur une souveraineté, sans que ses bénéficiaires aient à en payer le prix en terme de processus de légitimation, c'est-à-dire d'acquisition d'une légitimité. Bien évidemment la dématérialisation n'est pas la cause d'un tel mouvement, mais seulement - au sein de l'idéologie de la communication - l'une de ses conditions de possibilité.

Bref, aujourd'hui une critique de l'idéologie de la communication comme opération politique ne peut faire l'économie d'une critique de la dématérialisation.

\section{PASCAL ROBERT}

23. Pascal Robert, 1996, «Technologies de l'information sans frontières?, la fonction-frontière à l'épreuve des technologies de l'information et de la communication ", TIS, Vol. 8, n 3, Dunod, p. 213 à 242. Ce que confirme, comme malgré lui, nous l'avons vu, J.-P. Barlow lui-même. 